01

\title{
Моделирование системы определения движения технологической платформы по данным позиционирования ГЛОНАСС и измерениям ньютонометров
}

\author{
(C) A.C. Девятисильный, А.В. Шурыгин
}

Институт автоматики и процессов управления ДВО РАН, Владивосток, Россия

E-mail: devyatis@dvo.ru

Поступило в Редакцию 6 мая 2019г.

В окончательной редакции 6 мая 2019г.

Принято к публикации 7 июня 2019 г.

\begin{abstract}
Представлены модели реконструкции линейных и угловых параметров движения для бортовых встраиваемых систем ГЛОНАСС с двухпозиционным приемом данных и трехкомпонентным блоком ньютонометров в качестве измерителей удельных сил негравитационной природы. Существенным элементом конструкции математической модели является разработанная устойчивая процедура многократного численного дифференцирования, не зависящая от величины шага дискретизации задачи в условиях ограниченной точности вычислений и измерений. Приведены результаты численного эксперимента, верифицирующего исходные физические и математические представления для случая гиперзвукового движения.
\end{abstract}

Ключевые слова: ньютонометр, ГЛОНАСС, пульсар, гиперзвуковая скорость.

DOI: 10.21883/PJTF.2019.18.48231.17867

В настоящей работе рассмотрен вариант генерации системы определения движения (СОД) в случае, когда ее информационная база представлена измерениями вектора удельных сил негравитационный природы, или кажущегося ускорения [1], полученными с помощью ньютонометров [2], и темпоральными данными о координатах места подвижного объекта, доставляемыми навигационной спутниковой системой (НСC) типа ГЛОНАCC/GPS. Область применения таких СОД численно-аналитическое планирование, бортовое определение параметров движения и управление подвижными технологическими платформами (ТП) различного назначения и базирования (наземного, воздушного, космического).

Под „определением движения“ здесь понимается решение двух задач: во-первых, определение кинематических параметров траектории (траекторная задача) и, во-вторых, определение параметров ориентации ТП в пространстве (задача ориентации).

Предлагаемая СОД отличается от известных интегрированных инерциально-спутниковых систем [3-5] отсутствием в ней гироскопических измерителей абсолютной угловой скорости вращения ТП и методологией математического моделирования и решения обеих задач.

Учитывая форму геоида, в качестве его признанной опорной модели примем эллипсоид вращения (эллипсоид Клеро) [2,3]. В соответствии с этим и с учетом стандартов П3-90 (Россия) и WGS-89 (США) известным образом введем эллипсоидальную (геодезическую) систему отсчета с координатами $\{\varphi, \lambda, h\}$ - геодезические широта, долгота и высота над поверхностью эллипсоида.

В точке $O$, принадлежащей траектории и отождествляемой с подвижной ТП, разместим начало правого пря- моугольного координатного трехгранника $O x=O x_{1} x_{2} x_{3}$ с осью $O x_{3}$, направленной по нормали к поверхности эллипсоида, и осями $O x_{1}$ и $O x_{2}$, ориентированными на географические Восток и Север соответственно.

Обратимся теперь к кинематике, или к „геометрии движения“ $[1]$, точки $O$ и обозначим через $\mathbf{v}=\left(v_{1}, v_{2}, v_{3}\right)^{T}$ вектор ее линейной скорости движения относительно твердой Земли, а через $\boldsymbol{\omega}=\left(\omega_{1}, \omega_{2}, \omega_{3}\right)^{T}-$ вектор угловой скорости вращения трехгранника $O x$, обусловленной криволинейностью траектории. Оба вектора рассматриваются в проекциях на оси $O x$. Между координатами $\{\varphi, \lambda, h\}$ и скоростями достаточно просто установить следующие соотношения:

$$
\begin{gathered}
\omega_{1}=-\dot{\varphi}, \quad \omega_{2}=\dot{\lambda} \cos \varphi, \\
\omega_{3}=\dot{\lambda} \sin \varphi, \quad \dot{\varphi}=\frac{v_{2}}{r_{2}}, \quad \dot{\lambda}=\frac{v_{1}}{r_{1} \cos \varphi}, \quad v_{3}=\dot{r}_{\psi},
\end{gathered}
$$

где

$$
\begin{gathered}
r_{1}=a /\left(1-e^{2} \sin ^{2} \varphi\right)^{1 / 2}+h, \\
r_{2}=a\left(1-e^{2}\right) /\left(1-e^{2} \sin ^{2} \varphi\right)^{3 / 2}+h
\end{gathered}
$$

- радиусы кривизны двух взаимно перпендикулярных главных нормалей сечений поверхности $h=$ const, проходящих через оси $O x_{1}$ (сечение, касательное к параллели) и $O x_{2}$ (меридиональное сечение); $a$ и $e$ - значения большой полуоси и эксцентриситета используемой модели земного эллипсоида; $r_{\psi}=\left(r_{1}^{-1} \sin ^{2} \psi+r_{2}^{-1} \cos ^{2} \psi\right)^{-1}-$ радиус кривизны нормального сечения эллипсоида при $h=$ const в точке $O$, проходящего через вектор-проекцию $\mathbf{v}_{p}$ вектора $\mathbf{v}$ на плоскость $O y_{1} y_{2}[2] ; \psi-$ путевой угол движения, отсчитываемый по ходу часовой стрелки от оси $O y_{2}$ к оси $O y_{1}$, так что $v_{1}=v_{p} \sin \psi, v_{2}=v_{p} \cos \psi, v_{p}=\left|\mathbf{v}_{p}\right|$. 
Под решением траекторной задачи далее понимается решение задачи оценки значений и их производных до порядка $n-1$ параметров $\varphi, \lambda, h, r_{1}, r_{2}, r_{\psi}, v_{1}, v_{2}$, $v_{3}$ и т.д. При этом математическая модель такой общей задачи рассматривается как совокупность моделей частных обратных задач вида „состояния-измерения“ порядка $n$, где каждая из частных моделей ассоциируется с некоторой функцией времени $\eta(t)$, аппроксимируемой моделью „состояний“ вида $\eta(t)=\eta_{1}$, $\dot{\eta}_{1}=\dot{\eta}_{2}=\cdots=\dot{\eta}_{n}=0$, по ее темпорально измеряемым с инструментальными погрешностями значениям $\eta\left(t_{k}\right)$, $k=0,1,2, \ldots ; t_{k+1}=t_{k}+\tau, \tau=$ const. Как видно, каждая частная модель „состояний“ - это система линейных дифференциальных уравнений первого порядка с простейшей одноклеточной жордановой (с нулевыми диагональными элементами) матрицей [6] в качестве матрицы связи, имеющей индекс нильпотентности, равный $n$. Естественное обращение к такой модели существенно упростило дискретизацию задачи по времени и реализацию нейросетевого алгоритма динамического псевдообращения $[7,8]$ калмановского типа с ядерным механизмом настройки [8] на фоне преодоления проблемы разрешимости задачи при больших значениях $n$ и малых $\tau$ в условиях конечной точности вычислений и измерений [9].

Полагая далее, что траекторная задача решена [9], обратимся к динамике теперь уже материализованной точки $O$, т.е. к уравнениям Ньютона, которые с учетом вращения Земли с угловой скоростью $\mathbf{u}=\left(u_{1}, u_{2}, u_{3}\right)^{T}$ в осях трехгранника $O x$ представимы в следующем виде:

$$
D_{i j} V_{j}=g_{i}+f_{j}, \quad V_{j}(0)=V_{0, i},
$$

где $D_{i j}=\delta_{i j} d / d t+e_{i k j}\left(\omega_{k}+u_{k}\right)$ - оператор абсолютной производной; $\delta_{i j}-$ символ Кронекера; $e_{i k j}-$ символ Леви-Чивита $(i, k, j=1,2,3$; по повторяющимся индексам производится суммирование); $u_{1}=0$, $u_{2}=u \cos \varphi, \quad u_{3}=u \sin \varphi ; \quad \mathbf{V}=\left(V_{1}, V_{2}, V_{3}\right)^{T}-$ вектор абсолютной скорости, причем $V_{1}=v_{1}+u_{2} r_{1}, V_{2}=v_{2}$, $V_{3}=v_{3} ; \mathbf{f}=\left(f_{1}, f_{2}, f_{3}\right)^{T}$ - вектор кажущегося ускорения; $\mathbf{g}=\left(g_{1}, g_{2}, g_{3}\right)^{T}-$ напряженность гравитационного поля Земли, известного с достаточной точностью.

Разрешая уравнение (2) относительно f покомпонентно и принимая во внимание (1), получаем

$$
\begin{gathered}
f_{1}=\dot{v}_{1}+\frac{v_{1}}{r_{1}}\left(v_{3}-v_{2} \operatorname{tg} \varphi\right)-v_{2} u_{3}\left(\frac{r_{1}}{r_{2}}+1\right) \\
+v_{3} u_{2}+u_{2} \dot{r}_{1}-g_{1}, \\
f_{2}=\dot{v}_{2}+\frac{v_{1}^{2}}{r_{1}} \operatorname{tg} \varphi+\frac{v_{2} u_{3}}{r_{2}}+2 v_{1} u_{3}+u_{2} u_{3} r_{1}-g_{2}, \\
f_{3}=\dot{v}_{3}-\frac{v_{1}^{2}}{r_{1}}+\frac{v_{2}^{2}}{r_{2}}-2 v_{1} u_{2}-u_{2}^{2} r_{1}-g_{3} .
\end{gathered}
$$

С учетом того, что все значения переменных в правой части (3) известны благодаря интерпретации данных спутникового позиционирования ТП, т.е. в результате решения траекторной задачи, вектор $\mathbf{f}$ также может быть вычислен. Таким образом, устанавливается и каузальность траектории.

Введем жестко связанный с подвижной ТП правый координатный трехгранник, в осях которого могут производиться векторные измерения, - приборный трехгранник $\mathrm{Oy}=\mathrm{Oy}_{1} \mathrm{Oy}_{2} \mathrm{Oy}_{3}$, в идеале совпадающий с трехгранником $O x$, но реально связанный с ним матрицей ориентации $\mathbf{A}=\left(a_{i j}\right)(i, j=1,2,3)$, так что $y_{i}=a_{i j} x_{j}$ и $x_{j}=a_{j i} y_{i}$. Суть задачи определения ориентации ТП в физическом пространстве состоит в оценке матрицы А или значений трех углов последовательного вращения трехгранника $O y$ из состояния $O y \equiv O x$ в текущее состояние. Учитывая, что под ТП здесь понимается объект искусственного происхождения, целесообразно считать, что оси трехгранника $O y$ совпадают со строительными осями ТП, и ввести углы Эйлера-Крылова - углы курca $(\alpha)$, крена $(\beta)$ и тангажа $(\theta)$, соответствующие парциальным вращениям относительно осей $O y_{3}, O y_{2}, O y_{1}$.

Для определения значений девяти элементов (направляющих косинусов) матрицы А (или трех углов Эйлера-Крылова), вообще говоря, достаточно найти систему двух неколлинеарных векторов, известных своими проекциями как в $O x$, так и в $O y$. В качестве двух таких векторов могут быть взяты орты известных звезд, пульсаров или иных объектов. Корректность решения проблемы в таком случае обусловливается возможностью пополнения системы двух векторов третьим, образуемым векторным произведением известных двух, и, таким образом, линейной независимостью этих трех векторов. В предельном случае, когда наблюдению в $O x$ и $O y$ доступен только один вектор, определить ориентацию $O y$ относительно $O x$ можно только с точностью до поворота $O y$ относительно $O x$ вокруг единственного вектора как оси.

Возможность вычисления $\mathbf{f}$, демонстрируемая системой уравнений (3), обращает внимание на методологически другую возможность - непосредственное измерение проекций $\mathbf{f}$ в трехграннике $O y$ с помощью тройки линейных ньютонометров; таким образом решается проблема выбора первого из двух требуемых векторов. Однако существенно отметить, что, оставаясь в рамках анонсируемой в настоящей работе задачи комплексирования измерений геодезических координат места объекта с помощью НСС и измерений ньютонометров, также можно строго решить проблему и второго вектора, но только в случае двухпозиционного (и более) бортового приема данных НСС. Вместе с тем, как показывают вычислительные эксперименты, и при однопозиционном приеме возможны вполне допустимые приближенные оценки углов, если отождествлять угол $\alpha$ с путевым углом $\psi$ в рамках псевдоизмерения $\alpha=-\psi+\delta \alpha$, когда $\delta \alpha$ выступает в качестве небольшой по величине методологической погрешности, обусловленной взаимодействием подвижной ТП с внешней средой. В таком случае методом последовательных приближений достаточно просто и в пределах шага дискретизации $\tau$ решается система 

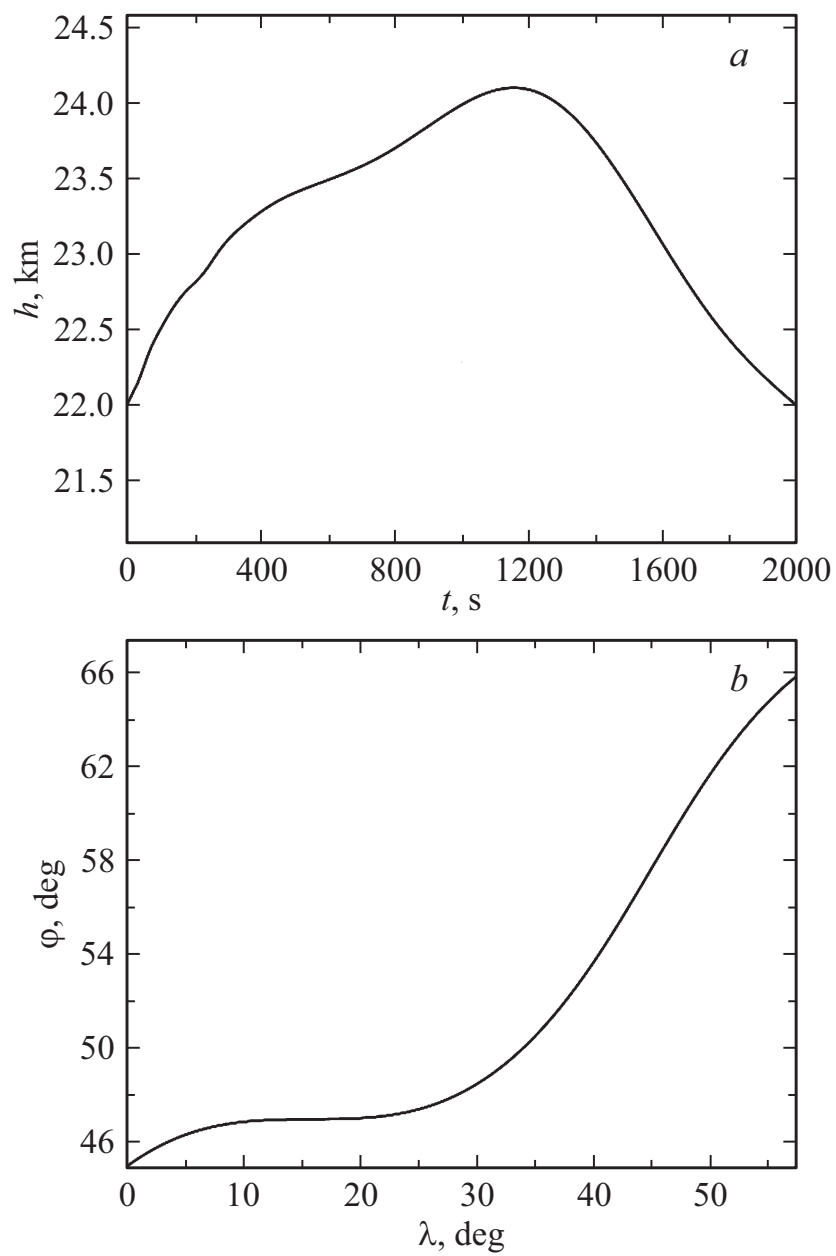

Рис. 1. Элементы траектории движения: высота $(a)$, широта и долгота $(b)$.

уравнений $\mathbf{f}_{y}=\mathbf{A f}$, где $\mathbf{f}_{y}$ - вектор силы f, измеряемой ньютонометрами, т.е. задача точечного оценивания углов $\beta$ и $\theta$. Здесь же, в пределах этого же шага, оценивается необходимое число производных углов $\alpha$, $\beta, \theta$ и таким образом реализуется возможность оценки в трехграннике $O y$ вектора угловой скорости собственного вращения $\mathbf{q}=\left(q_{1}, q_{2}, q_{3}\right)^{T}$, где $q_{1}=\dot{\theta}+\dot{\alpha} \sin \beta$, $q_{2}=\dot{\beta} \cos \theta+\dot{\alpha} \sin \theta \cos \beta, q_{3}=\dot{\alpha} \cos \theta \sin \beta-\dot{\beta} \sin \theta$.

Подобным же образом вычисляется и вся тройка углов Эйлера-Крылова, т. е. $\{\alpha, \beta, \theta\}$, и дополнительно угол курсового дрейфа $\delta \alpha=\psi-\alpha$, если на борту ТП осуществляется двухпозиционный прием спутниковой навигационной информации и решается система уравнений $\left\{\mathbf{f}_{y}=\mathbf{A f}, \mathbf{l}_{y}=\mathbf{A} \mathbf{l}\right\}$, где $\mathbf{l}_{y}-$ технологический (известный) вектор места второго приемника $\mathrm{HCC}$ в $O y$, а 1 - этот же вектор в $O x$, вычисляемый по данным его позиционирования НСС. Очевидно, возможен и отказ от исходной декларируемой бортовой системы измерителей и пополнения ее астровизирами известных звезд или пульсаров.

В общем случае, если вектор q определен, открывается возможность и для оценки абсолютной угловой скорости ТП $\mathbf{p}=\left(p_{1}, p_{2}, p_{3}\right)^{T}$ в проекциях на оси $O y$, а именно $\mathbf{p}=\mathbf{A}(\boldsymbol{\omega}+\mathbf{u})_{x}+\mathbf{q}$, где индекс $x$ указывает на то, что вектор $\boldsymbol{\omega}+\mathbf{u}$ представлен своими проекциями в трехграннике $O x$. Таким образом, по сути, реализуется безгироскопный датчик угловых скоростей, что с учетом возможности качественного дифференцирования вектора p открывает путь для оценки главного момента сил - вектора $\mathbf{m}$, обусловливающего совместно c вектором f движение реальной ТП. Действительно, $m_{i}=J_{i j} \dot{p}_{j}+\left(e_{i k j} p_{k}\right) J_{j s} p_{s}$, где $\mathbf{J}=\left(J_{j s}\right)$ - известный тензор инерции ТП, $i, j, k, s=1,2,3$.

На рис. 1 представлена траектория ТП, движущейся с гиперзвуковой скоростью $v=3560 \mathrm{~m} / \mathrm{s}(12 \mathrm{M}$, $\mathrm{M}_{h=20 \mathrm{~km}} \approx 298 \mathrm{~m} / \mathrm{s}$ ). Данные позиционирования поступают с шагом $\tau=0.2 \mathrm{~s}$, содержат погрешности со среднеквадратическими значениями (СКЗ) $\sigma_{\varphi}=\sigma_{\lambda}=\sigma_{h}=1.5 \mathrm{~m}$ и временами корреляции $\tau_{c}=4 \mathrm{~s}$. На рис. 2, $a$ представлены оценки сил, выполняемые по формулам (3); СК3 погрешностей оценивания $\sigma_{f_{1}}=\sigma_{f_{2}}=\sigma_{f_{3}}=0.006 \mathrm{~m} / \mathrm{s}^{2}$. На рис. $2, b$ представлены оценки углов $\alpha, \beta, \theta$; СКЗ погрешностей оценивания $\sigma_{\alpha}=\sigma_{\beta}=\sigma_{\theta}=0.01^{\circ}$. При этом погрешности шумов ньютонометров характеризуются СКЗ $\sigma_{f_{1}}=\sigma_{f_{2}}=\sigma_{f_{3}}=0.01 \mathrm{~m} / \mathrm{s}^{2}$ и временами корреля-
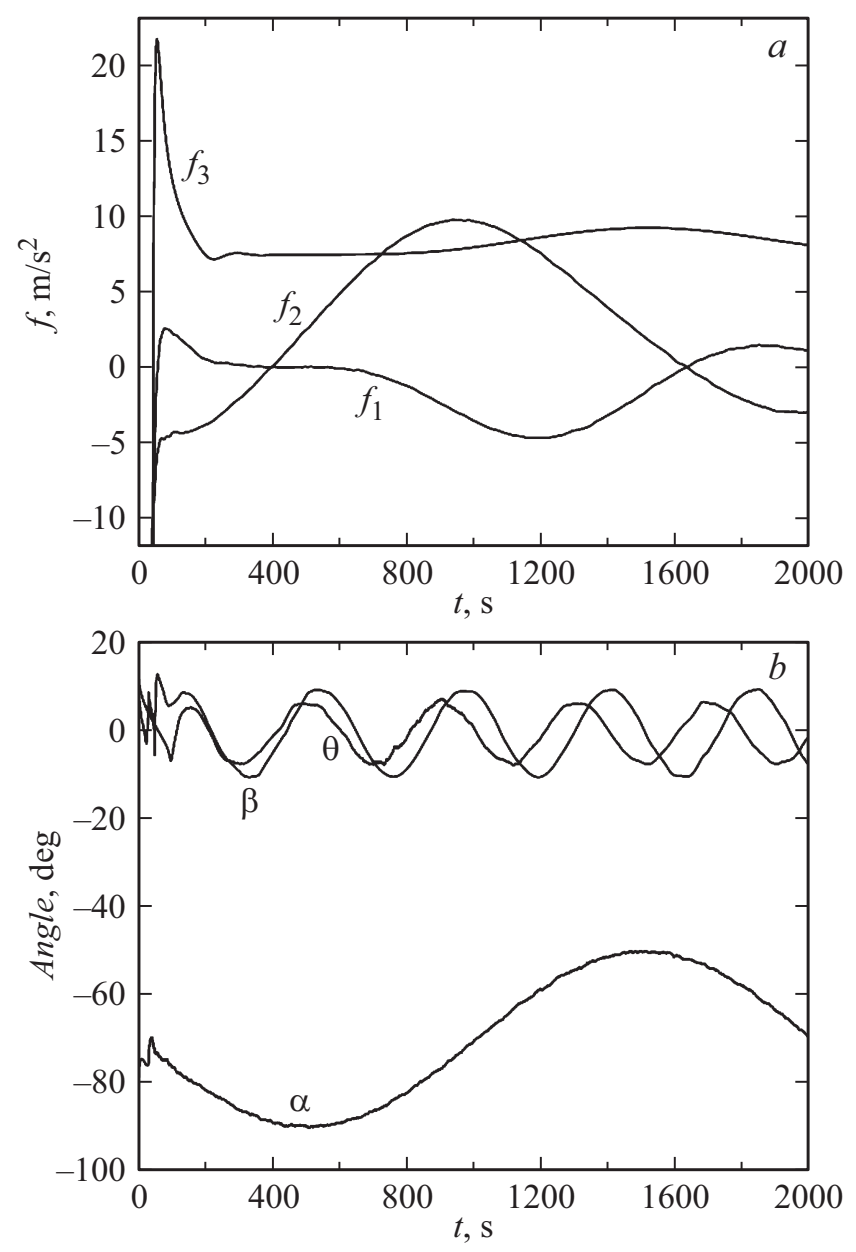

Рис. 2. Оценки удельных сил (a) и углов Эйлера-Крылова $(b)$. 
ции $\tau_{c}=1.5 \mathrm{~s}$; место второго приемника $\mathrm{HCC}$ в $O y$ : $\mathbf{y}=(0,4 \mathrm{~m}, 0)^{T}$.

Таким образом, как подтверждается результатами численного исследования, предложенная модель безгироскопной гибридной инерциально-спутниковой системы определения движения обладает высокой эффективностью решения как траекторной задачи, так и задачи пространственной ориентации и актуальна для управления высокоскоростными объектами.

\section{Конфликт интересов}

Авторы заявляют, что у них нет конфликта интересов.

\section{Список литературы}

[1] Иилинский А.Ю. Классическая механика и силы инерции. М.: Едиториал УРСС, 2018. 320 с.

[2] Андреев В.Д. Теория инерциальной навигации. Корректируемые системы. М.: Наука, 1967. 648 с.

[3] Перов А.И., Харисов В.Н. ГЛОНАСС. Принципы построения и функционирования. М.: Радиотехника, 2010. 800 с.

[4] Groves P. Challenges of integrated navigation // Proc. of the 31st Int. Technical Meeting of the satellite division of the institute of navigation. Miami, 2018. P. 3237-3264.

[5] Mahboub V., Mohammadi D. // J. Navigation. 2018. V. 71. N 4. P. $971-988$.

[6] Хорн Р., Джсонсон Ч. Матричный анализ. М.: Мир, 1989. $656 \mathrm{c}$.

[7] Осипов Ю.С., Кряэкемский А.В. // Вестн. РАН. 2006. Т. 76. № 7. С. 615-624.

[8] Девятисильный А.С. // ЖТФ. 2015. Т. 85. В. 10. С. 5-8.

[9] Девятисильный А.С., Шурыгин А.В. // Геодезия и картография. 2018. Т. 79. В. 1. С. 47-51. 